\title{
Dynamic Modeling of Pedestrian Legform and Prediction of Pedestrian Injuries
}

\author{
Shigeru Ogawa, Soma Usui, Yudai Kawaguchi \\ Department of Mechanical Engineering, National Institute of Technology, Kure College, Hiroshima, Japan \\ Email: ogawa@kure-nct.ac.jp
}

How to cite this paper: Ogawa, S., Usui, S. and Kawaguchi, Y. (2017) Dynamic Modeling of Pedestrian Legform and Prediction of Pedestrian Injuries. Open Journal of Modelling and Simulation, 5, 241-252. https://doi.org/10.4236/ojmsi.2017.54018

Received: June 19, 2017

Accepted: October 28, 2017

Published: October 31, 2017

Copyright $\odot 2017$ by authors and Scientific Research Publishing Inc. This work is licensed under the Creative Commons Attribution International License (CC BY 4.0).

http://creativecommons.org/licenses/by/4.0/

(c) (i) Open Access

\begin{abstract}
Decreasing the death toll of pedestrians in traffic accidents is one of the most urgent tasks to be solved all over the world. This paper describes the prediction of pedestrian injuries for the TRL legform impactor using MATLAB. The TRL legform impactor consists of three parts: a femur, a tibia, and a ligament connecting them. The impactor was physically modelled with springs, dampers and two masses as a dynamic model. The impactor behaves in a translational and rotational motion during the collision with a vehicle. The behavior of the impactor during the crash event was captured by a high speed camera and is regarded as the four-degree-of-freedom system in terms of translational and rotational motions. Pedestrian injuries are evaluated by three physical quantities indexes: the acceleration of the tibia, both the displacement and the bending angle between the femur and the tibia. The physical model for the impactor was expressed mathematically by differential equations. In the case of modelling, the ligament connecting both the femur and the tibia in particular plays an important role. Shear forces were applied to the ligament in translational motions and the bending moments in rotational motion. Differential equations were expressed in the form of a state equation and an output equation by MATLAB. Numerical solutions were obtained by a block diagram with Simulink. As a result, it was found that the predicted injuries agree quite well with their experimented data in terms of acceleration, displacement, and the bending angle mentioned above.
\end{abstract}

\section{Keywords}

Dynamic Model, Spring-Mass Model, MATLAB, Pedestrian Injuries, TRL Legform Impactor

\section{Introduction}

Pedestrian protection test was originally proposed by European Enhanced Ve- 
hicle-safety Committee: EEVC WG 10 in 1994 and has been discussed by EEVC WG17 and International Organization for Standardization: ISO, and International Harmonized Research Activities: IHRA [1]-[7]. The recent number of casualties by traffic accidents in Japan tends to decrease owing to the progress of safety technology of automobiles and environmental improvement of roads. However, since 2009 fatalities among people killed while walking on the streets have exceeded those while driving and the percentage in total death toll are increasing as shown in Figure 1. Legform impact tests shown in Figure 2 have been conducted since 2011 in JNCAP (Japan New Car Assessment Program). Since leg injuries are the most common injuries in pedestrian accidents, it is urgent tasks to minimalize leg injuries. It therefore follows that the study is to seek for safety concept body designed to mitigate injuries to pedestrians. For the first step, the paper describes to model the Transport Research Laboratory (TRL) legform impactor and to solve the equations of motion using commercial software MATLAB.

\section{Dynamic Model of TRL Legform Impactor}

\subsection{Mathematical Modeling}

Figure 3 shows the schematic diagram of TRL impactor which consists of the

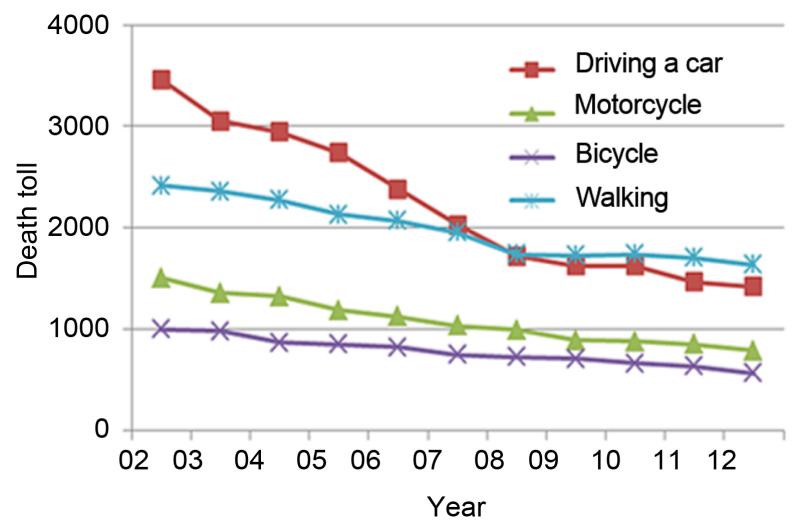

Figure 1. Change of death toll by traffic accidents in Japan.

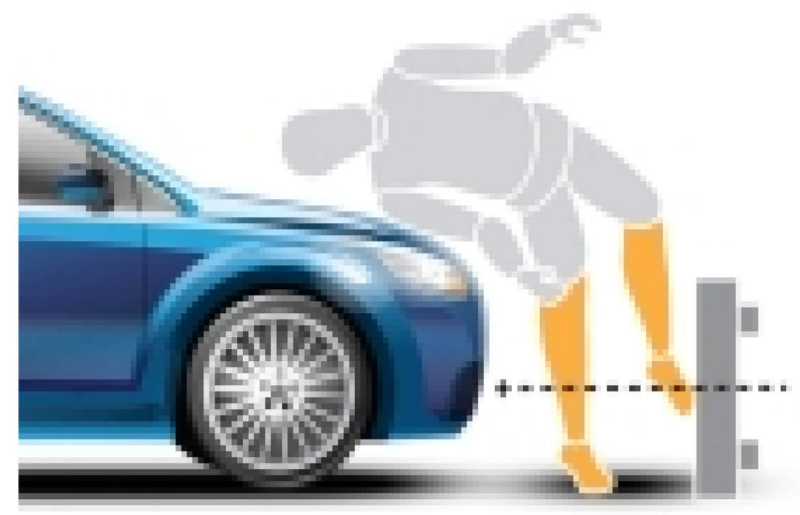

Figure 2. Schematic image of legform impacte test. 

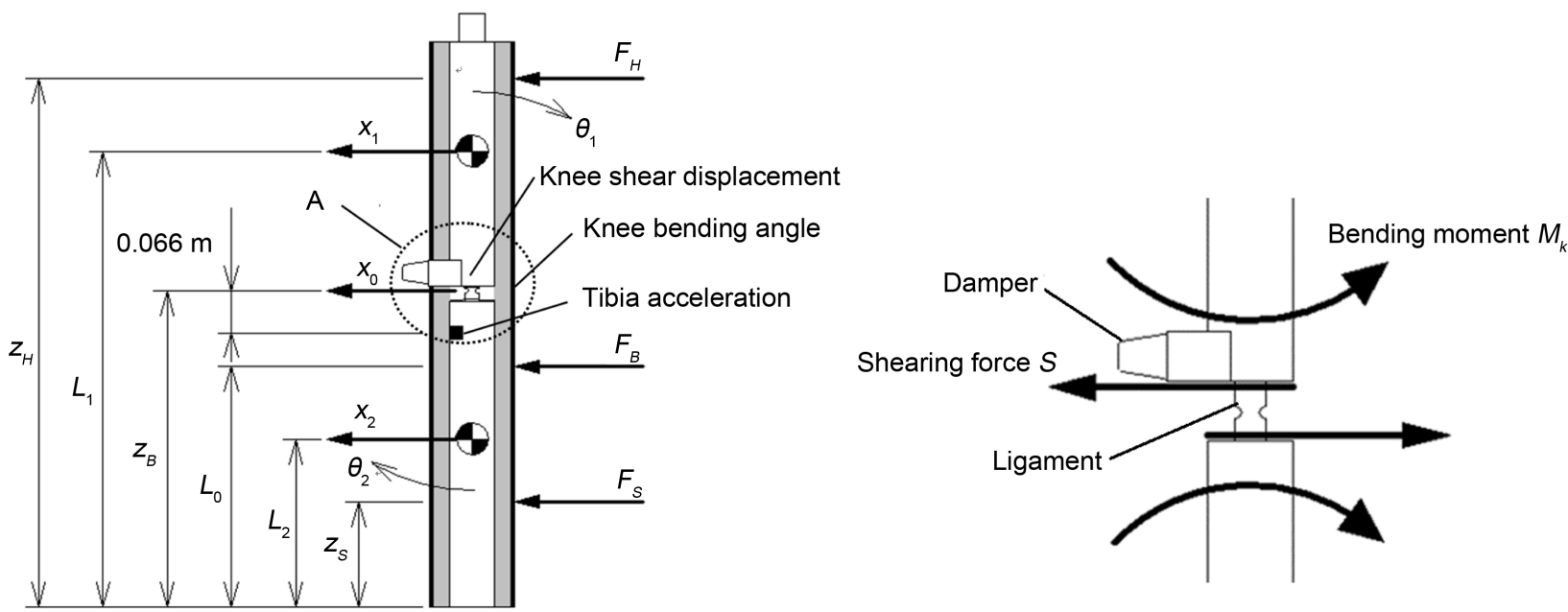

Figure 3. Schematic diagram of an impactor and its enlarged schema of ligament.

upper legform and the lower legform and a ligament connecting them including enlarged schema of ligament. TRL impactor mainly consists of two stiff metal tubes, two deformable knee elements made of steel and a shear-spring system with a hydraulic damper. The two stiff metal tubes represent the femur and the tibia of a human leg. The deformable knee elements represent the human knee, specifically the ligaments, with the ability to withstand a certain bending. The metal "ligaments" are used to assess possible knee injuries. The shear-spring system simulates lateral shear displacement between femur and tibia at the knee level; the damper is necessary to limit vibrations caused by the mass of the shear-spring system. An accelerometer is used to indirectly measure the contact force applied to the tibia, representing a provisional assessment of the risk of bone fractures. For testing, the legform is covered with a $25 \mathrm{~mm}$ thick foam layer and a $6 \mathrm{~mm}$ neoprene skin, together representing the human's flesh and skin. TRL impactor is used to test cars to determine their potential to cause injury when they impact into pedestrians. The impactor is fired into a stationary car at speeds up to $40 \mathrm{~km} / \mathrm{h}$. The upper legform (femur) is its total length $=432 \mathrm{~mm}$, position of center of gravity $L_{1}=711 \mathrm{~mm}$, mass $m_{1}=8.6 \mathrm{~kg}$, inertia moment $I_{1}=$ $0.127 \mathrm{~kg} \cdot \mathrm{m}^{2}$, whereas the lower legform (tibia), is its total length $=494 \mathrm{~mm}$, position of center of gravity $L_{2}=261 \mathrm{~mm}$, mass $m_{2}=4.8 \mathrm{~kg}$, inertia moment $I_{2}=$ $0.120 \mathrm{~kg} \cdot \mathrm{m}^{2}$. Their cylindrical shapes have $70 \mathrm{~mm}$ in diameter. The ligament connecting the femur and the tibia is elastically deformed by absorbing impact energy. In case of tests, the impactor is fully covered with foam cover. The measured items to be evaluated in NCAP are tibia acceleration $\alpha_{T}$, knee shearing displacement $\tau$, and knee bending angle $\theta$, which are employed as injury criteria for fracture of tibia, damages of cruciate ligament, and collateral ligament, respectively. Their maximum values are used as evaluation indices. In the typical case when the impactor collides with a vehicle, the impactor is subjected to contact forces from three portions of the vehicle; $F_{H}$ at $z_{H}$ from a hood, $F_{B}$ at $z_{B}$ from a bumper, and $F_{S}$ at $z_{S}$ from a spoiler. 


\subsection{State Equations of the System}

The behavior of the impactor during the crash event was captured by a high speed camera. As a result, it was found that the impactor is regarded as the four-degree-of-freedom system in terms of the translational and the rotational motions. The impactor is physically modelled with springs, dampers and two masses as a dynamic model. The impactor behaves in translational and rotational motions during the collision with a vehicle. The equations of the translational motions with respect to the femur and the tibia are expressed as (1) and (2), respectively:

$$
\begin{gathered}
m_{1} \ddot{x}_{1}=F_{H}+S, \\
m_{2} \ddot{x}_{2}=F_{B}+F_{S}-S,
\end{gathered}
$$

where $x_{1}$ and $x_{2}$ show the coordinates of the gravity center of the femur and tibia respectively and $F_{H}$ is the force applied to the femur from the hood, $F_{B}$ and $F_{S}$ are the forces applied to the tibia from a bumper and a spoiler, respectively, whereas $S$ indicates shearing forces exerted on the ligament in the opposite direction each other as shown in Figure 3.

The equations of rotational motions with respect to the femur and the tibia are given as (3) and (4), respectively (see Figure 3):

$$
\begin{gathered}
I_{1} \ddot{\theta}_{1}=T_{H}+S L_{10}-K, \\
I_{2} \ddot{\theta}_{2}=T_{B}+T_{S}+S L_{20}+K,
\end{gathered}
$$

where $\theta_{1}$ and $\theta_{2}$ are rotational angles of the femur and the tibia, respectively and $L_{10}=L_{1}-L_{0}, L_{20}=L_{0}-L_{20}$.

Shearing forces $S$ are described as the sum of spring forces proportional to shearing displacements and viscous damping forces proportional to shearing velocity as in (5):

$$
S=-k_{s}\left[\left(x_{1}+L_{10} \theta_{1}\right)-\left(x_{2}-L_{20} \theta_{2}\right)\right]-c_{s}\left[\left(\dot{x}_{1}+L_{10} \dot{\theta}_{1}\right)-\left(\dot{x}_{2}-L_{20} \dot{\theta}_{2}\right)\right],
$$

where $k_{s}$ and $c_{s}$ are shearing linear elastic stiffness coefficient and shearing linear viscous damping coefficient, respectively.

Figure 4 shows the relationship between shearing displacements and static loads obtained by the static bending moment test. The spring constant was determined as $k_{s}=571,429(\mathrm{~N} / \mathrm{m})$ so that the related curve can be placed between the upper and the lower limit, which complies with test procedures. On the other hand the shearing viscous damping coefficient is analytically obtained by MATLAB as $c_{s}=750 \mathrm{Ns} / \mathrm{m}$ as described later.

The terms on the right hand of (3) and (4) express the moments, where $T_{H}, T_{B}$, $T_{\mathcal{S}}$ and $K$ are defined as follows:

$$
\begin{aligned}
& T_{H}=F_{H}\left(L_{1}-z_{H}\right), \\
& T_{B}=F_{B}\left(L_{2}-z_{B}\right), \\
& T_{S}=F_{S}\left(L_{2}-z_{S}\right),
\end{aligned}
$$




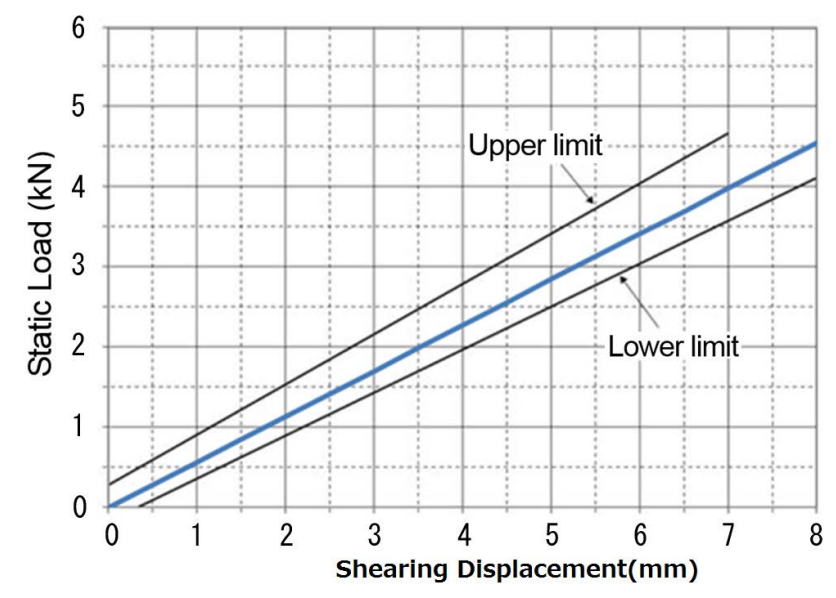

Figure 4. Relationship between shearing displacement and static load in static bending moment test.

$$
K=M_{K}+C_{K},
$$

where just as described in shearing force $S$, bending moment $K$ is expressed as the sum of $M_{k}$ induced by the function of rotational angle $\theta$ and $C_{k}$ induced by angular velocity. $C_{m}$ is rotational viscous damping coefficient determined as 33 $(\mathrm{Nm} / \mathrm{rad})$ by MATLAB.

$$
\begin{gathered}
M_{k}=\left(10+\frac{75}{\theta^{2}+\frac{0.05}{\theta^{2}}}+\frac{600}{\theta^{2}+\frac{2.8}{\theta^{2}}}+\frac{1200}{\theta^{2}+\frac{28}{\theta^{2}}}+\frac{500}{\theta^{2}+\frac{700}{\theta^{2}}}\right. \\
\left.-\frac{75}{\theta^{2}+\frac{10}{\theta^{2}}}-\frac{600}{\theta^{2}+\frac{20}{\theta^{2}}}-\frac{1200}{\theta^{2}+\frac{150}{\theta^{2}}}-\frac{500}{\theta^{2}+\frac{150}{\theta^{2}}}\right) \times \theta \\
C_{k}=C_{m} \dot{\theta}=C_{m}\left(\dot{\theta}_{1}-\dot{\theta}_{2}\right)
\end{gathered}
$$

here $M_{k}$ consists of two regions, an elastically deformed region and a plastically deformed region. Figure 5 shows the characteristics of the ligament which has elastic deformation region between $\theta=0$ and 2 degrees whereas plastic deformation one over 2 degrees. The figure also shows the corridor surrounded by two black lines, the lower and the upper limit. For the elastic region $M_{k}$ increases linearly until 2 degrees in the corridor. Over 2 degrees in the plastic region $M_{k}$ is expressed as polynomial of $\theta$, and $M_{K}=10 \theta$ as $\theta$ becomes infinity.

Equations of motion from (1) to (4) can be expressed in matrix form.

$$
M \ddot{x}+C \dot{x}+K x=U F
$$

here, $M, C, K$, denote the mass, damping and stiffness matrices, and $x, F$ are displacement vector and force vector, respectively:

$$
x=\left(\begin{array}{l}
x_{1} \\
x_{2} \\
\theta_{1} \\
\theta_{2}
\end{array}\right), M=\left(\begin{array}{cccc}
m_{1} & 0 & 0 & 0 \\
0 & m_{2} & 0 & 0 \\
0 & 0 & I_{1} & 0 \\
0 & 0 & 0 & I_{2}
\end{array}\right)
$$




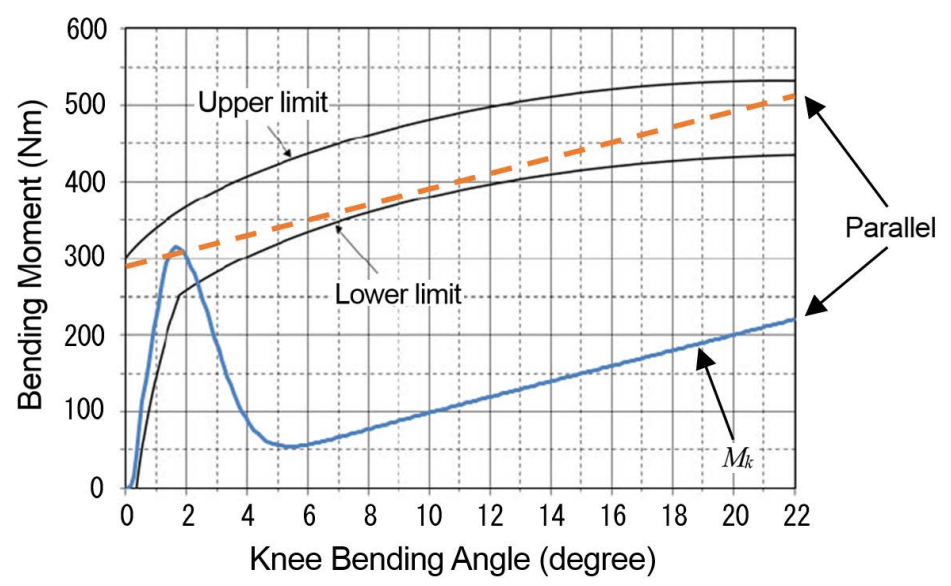

Figure 5. Relationship between knee bending angle and bending moment in static bending test.

$$
\begin{gathered}
C=C_{S}\left(\begin{array}{cccc}
1 & -1 & L_{10} & L_{20} \\
-1 & 1 & -L_{10} & -L_{20} \\
L_{10} & -L_{10} & L_{10}^{2} & L_{10} L_{20} \\
L_{20} & -L_{20} & L_{10} L_{20} & L_{20}^{2}
\end{array}\right), K=k_{s}\left(\begin{array}{cccc}
1 & -1 & L_{10} & L_{20} \\
-1 & 1 & -L_{10} & -L_{20} \\
L_{10} & -L_{10} & L_{10}^{2} & L_{10} L_{20} \\
L_{20} & -L_{20} & L_{10} L_{20} & L_{20}{ }^{2}
\end{array}\right) \\
U=\left(\begin{array}{ccccccc}
1 & 0 & 0 & 0 & 0 & 0 & 0 \\
0 & 1 & 1 & 0 & 0 & 0 & 0 \\
0 & 0 & 0 & 1 & 0 & 0 & -1 \\
0 & 0 & 0 & 0 & 1 & 1 & 1
\end{array}\right), F=\left(\begin{array}{c}
F_{H} \\
F_{B} \\
F_{S} \\
T_{H} \\
T_{B} \\
T_{S} \\
K
\end{array}\right)
\end{gathered}
$$

From (12) $\ddot{x}$ is given as follows:

$$
\ddot{x}=-M^{-1} C \dot{x}-M^{-1} K x+M^{-1} U F
$$

State Equation (20) is expressed with (17), (18), and (19):

$$
\begin{gathered}
X=X_{1}, \dot{X}_{1}=X_{2} \\
\dot{X}_{2}=-M^{-1} C X_{2}-M^{-1} K X_{1}+M^{-1} U F \\
\frac{\mathrm{d}}{\mathrm{d} t}\left(\begin{array}{c}
X_{2} \\
X_{1}
\end{array}\right)=\left(\begin{array}{cc}
-M^{-1} C & -M^{-1} K \\
1 & 0
\end{array}\right)\left(\begin{array}{c}
X_{2} \\
X_{1}
\end{array}\right)+\left(\begin{array}{c}
M^{-1} U \\
0
\end{array}\right) F \\
\dot{X}=A X+B F
\end{gathered}
$$

where

$$
A=\left(\begin{array}{cccccccc}
\multicolumn{3}{c}{-M^{-1} C} & & \multicolumn{3}{c}{-M^{-1} K} \\
1 & 0 & 0 & 0 & 0 & 0 & 0 & 0 \\
0 & 1 & 0 & 0 & 0 & 0 & 0 & 0 \\
0 & 0 & 1 & 0 & 0 & 0 & 0 & 0 \\
0 & 0 & 0 & 1 & 0 & 0 & 0 & 0
\end{array}\right), B=\left(\begin{array}{ccccccc}
0 \\
0 & 0 & 0 & 0 & 0 & 0 & 0 \\
0 & 0 & 0 & 0 & 0 & 0 & 0 \\
0 & 0 & 0 & 0 & 0 & 0 & 0 \\
0 & 0 & 0 & 0 & 0 & 0 & 0
\end{array}\right)
$$


Output equation is expressed as follows:

$$
Y=C X+D F
$$

where

$$
Y=\left(\begin{array}{l}
\ddot{x}_{2} \\
\ddot{\theta}_{2} \\
\dot{\theta}_{1} \\
\dot{\theta}_{2} \\
x_{1} \\
x_{2} \\
\theta_{1} \\
\theta_{2}
\end{array}\right), C=\left(\begin{array}{cccccccc}
\text { 2nd column of matrix } A \\
\text { 4th column of matrix } A \\
0 & 0 & 1 & 0 & 0 & 0 & 0 & 0 \\
0 & 0 & 0 & 1 & 0 & 0 & 0 & 0 \\
0 & 0 & 0 & 0 & 1 & 0 & 0 & 0 \\
0 & 0 & 0 & 0 & 0 & 1 & 0 & 0 \\
0 & 0 & 0 & 0 & 0 & 0 & 1 & 0 \\
0 & 0 & 0 & 0 & 0 & 0 & 0 & 1
\end{array}\right), D=\left(\begin{array}{ccccccc}
\text { 2nd column of matrix } B \\
\text { 4th column of matrix } B \\
0 & 0 & 0 & 0 & 0 & 0 & 0 \\
0 & 0 & 0 & 0 & 0 & 0 & 0 \\
0 & 0 & 0 & 0 & 0 & 0 & 0 \\
0 & 0 & 0 & 0 & 0 & 0 & 0 \\
0 & 0 & 0 & 0 & 0 & 0 & 0 \\
0 & 0 & 0 & 0 & 0 & 0 & 0
\end{array}\right)
$$

The standard TRL legform instrumentation includes two transducers to measure the relative rotation (bending angle) and relative translation (shear displacement) between the femur and the tibia. There is also an accelerometer fitted to the non-impact side of the tibia, close to the knee joint (66 $\mathrm{mm}$ below its knee). Figure 6 shows the schematic diagram of evaluation of pedestrian injuries. Based on output equation, pedestrian injuries by TRL legform are evaluated in terms of tibia acceleration $\alpha_{T}$ described in (24), knee shear displacement $\tau$ in (25), and knee bending angle $\theta$ in (26):

$$
\begin{gathered}
\alpha_{T}=\ddot{x}_{2}-\left(L_{0}-L_{2}-0.066\right) \ddot{\theta}_{2} \\
\tau=\left[x_{2}-\left(L_{0}-L_{2}\right) \theta_{2}\right]-\left[x_{1}+\left(L_{1}-L_{0}\right) \theta_{1}\right] \\
\theta=\theta_{1}-\theta_{2}
\end{gathered}
$$

\subsection{Solution by MATLAB}

MATLAB has embedded software called Simulink which provides an essential way to model, simulate and analyze the physical phenomena which are characterized by some inputs and outputs. The differential equations for TRL legform impactor were converted into state Equation (20) and output Equation (22). Two equations were implemented in Simulink as shown in Figure 7 using multipliers, summing, gain blocks, and subsequently fed into three integrators to obtain the states $\alpha_{T}, \tau$, and $\theta$.

\section{Test Procedures}

In order to validate the predicted injuries obtained by MATLAB, the experiment was conducted. Figure 8 shows the setup of TRL legform impactor. The impactor was fired at $40 \mathrm{~km} / \mathrm{s}$ into the energy absorption materials where load cells are incorporated on the rigid wall. Input forces, $F_{H} F_{B}$, and $F_{S}$ were measured by load cells. The measuring points are at $z_{H}=764 \mathrm{~mm}, z_{B}=404 \mathrm{~mm}, z_{S}=214 \mathrm{~mm}$. To simulate the characteristic of the actual vehicle bumper, the absorption material at middle position is approximately five and half times as hard as other two materials and protrudes $20 \mathrm{~mm}$ from them whereas the materials set at the hood and 

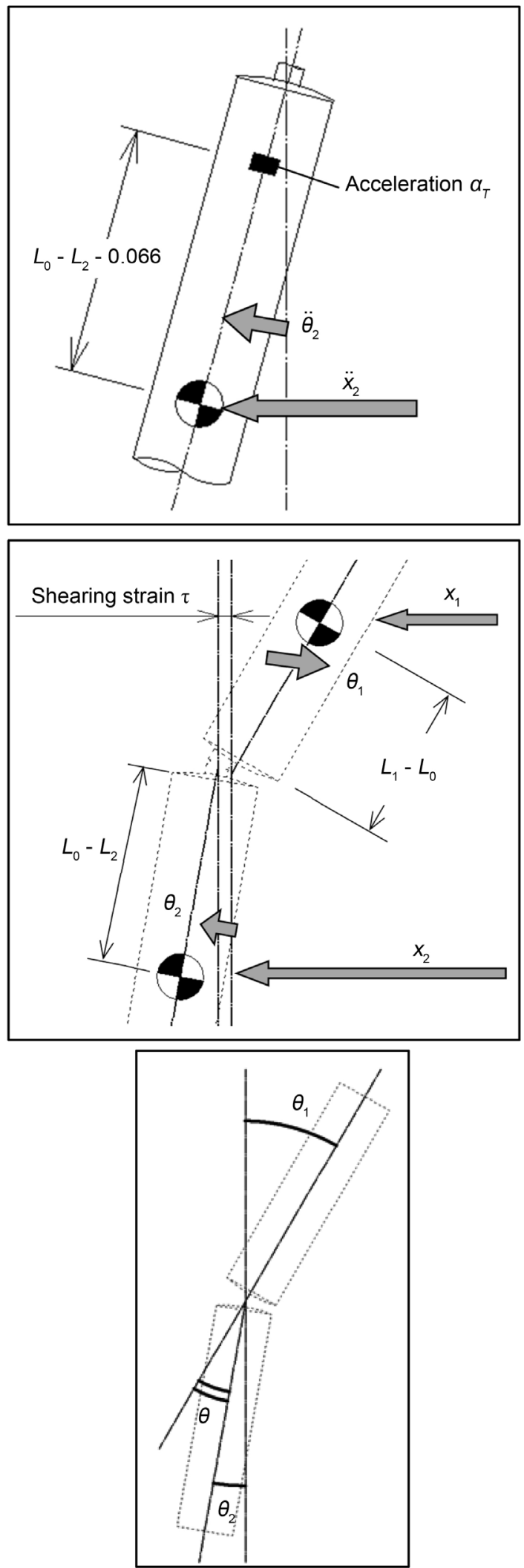

Figure 6. Evaluation of TRL legform impactor: Tibia acceleration (Upper), Shear displacement (Middle), and Bending angle (Lower). 


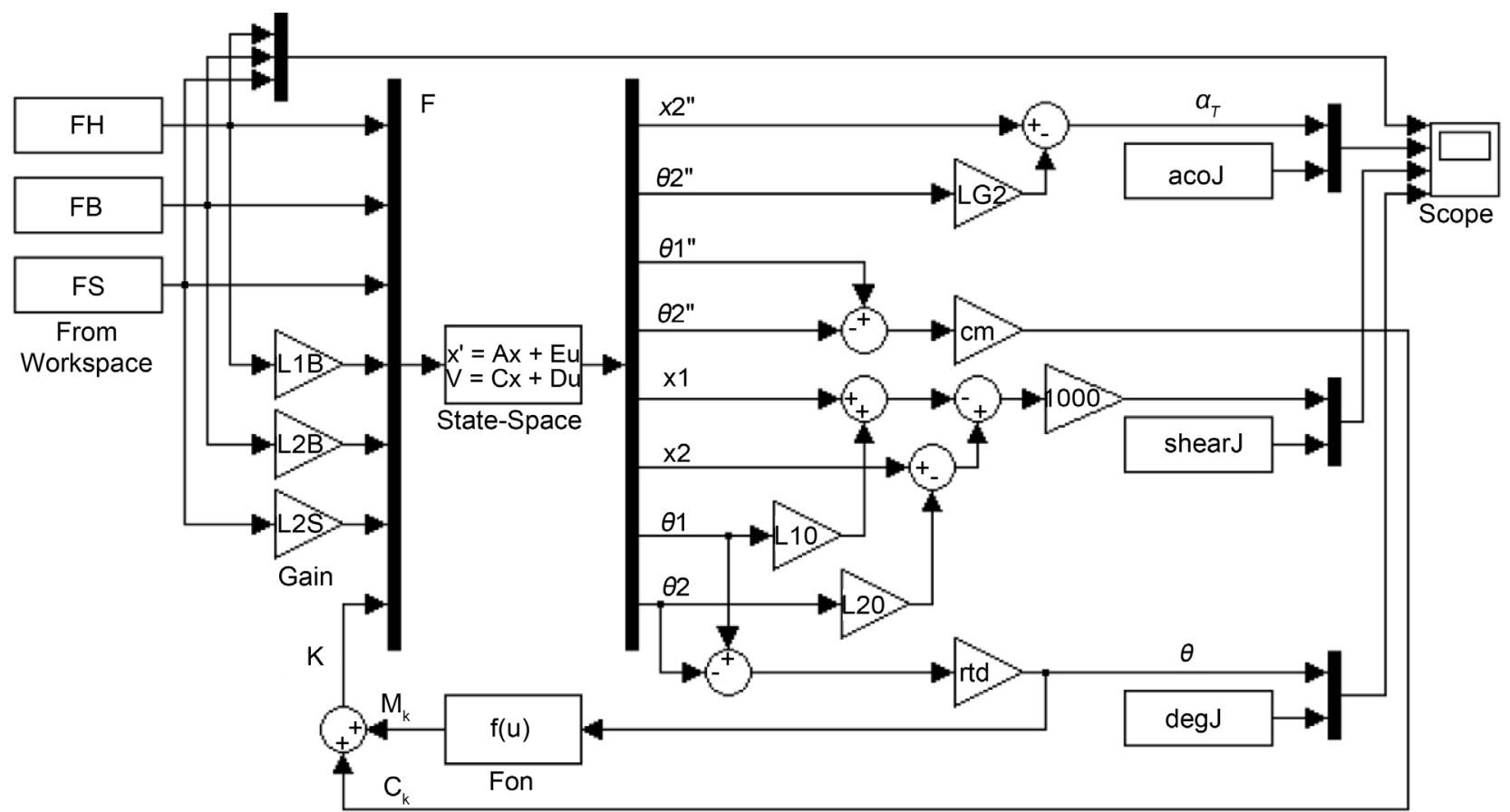

Figure 7. Block diagram for evaluation of pedestrian injuries: Tibia acceleration $\alpha_{T}$, Shear displacement $\tau$, Bending angle $\theta$.

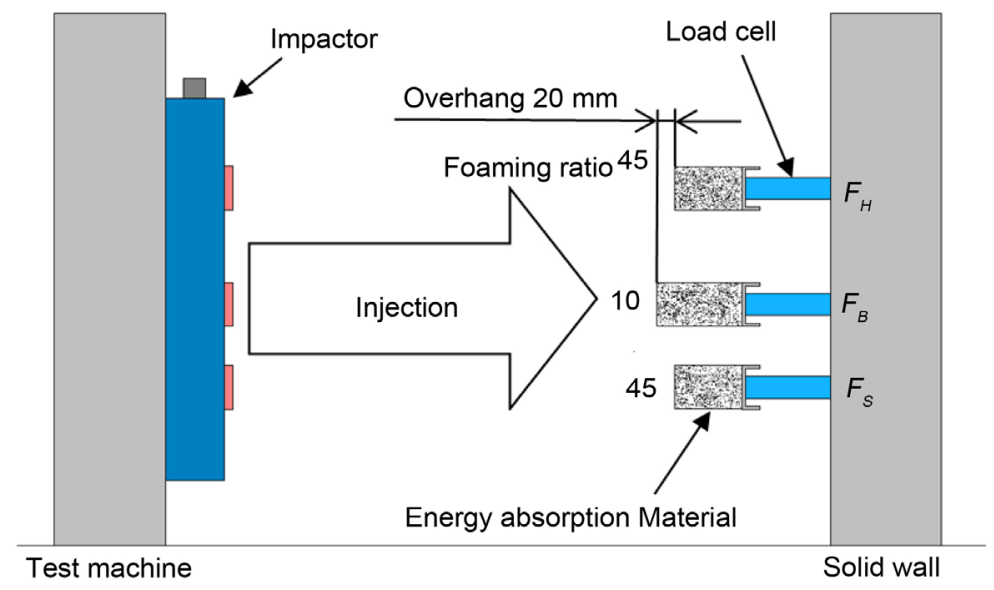

Figure 8. Test setup of TRL legform impactor.

the spoiler have the same hardness and the same position in the horizontal direction as shown in Figure 8.

Figure 9 depicts three input data obtained by the test. Reflecting the hardness and the position of energy absorption materials, the force $F_{B}$ is $9 \mathrm{kN}$ at peak value and four and half times as large as $F_{H}$ and $F_{S}$. Furthermore $F_{B}$ sharply increases and its duration of time lasts $20 \mathrm{~ms}$ whereas $F_{H}$ and $F_{S}$ are $2 \mathrm{kN}$ at peak value although the duration of time for $F_{H}$ is four times as long as $F_{S}$.

\section{Results and Discussions}

Forces obtained by the experiment shown in Figure 9 were used as input data in MATLAB Simulink to predict pedestrian injuries. Figure 10 shows the comparison 


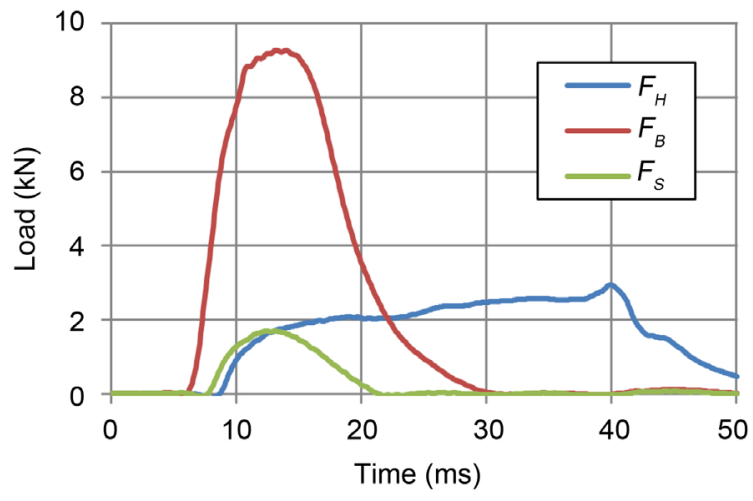

Figure 9. Input data for $F_{H}, F_{B}$ and $F_{S}$ of TRL legform impactor.

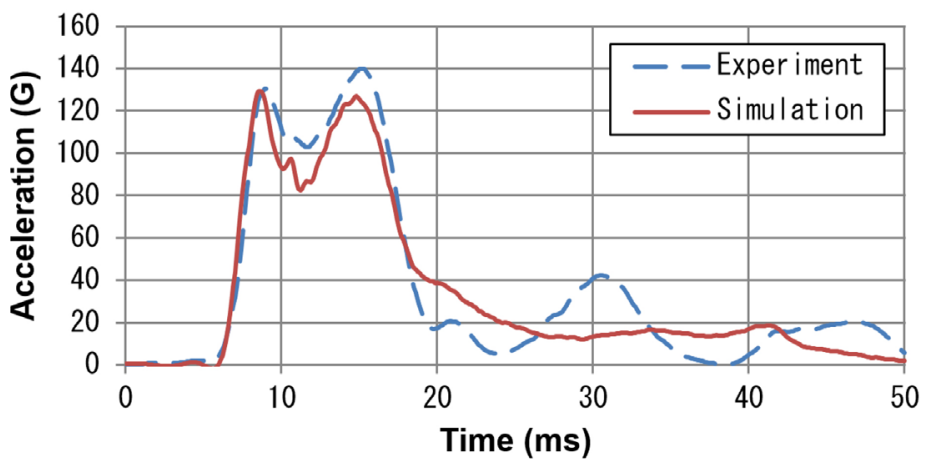

(a)

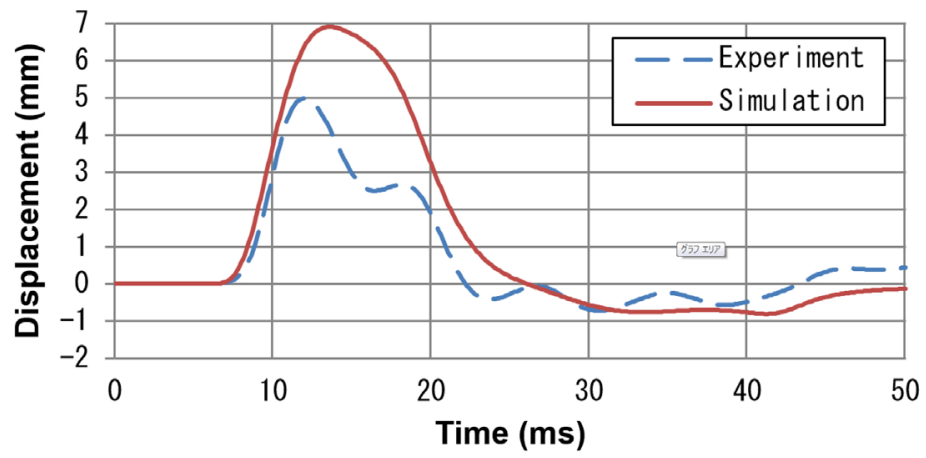

(b)

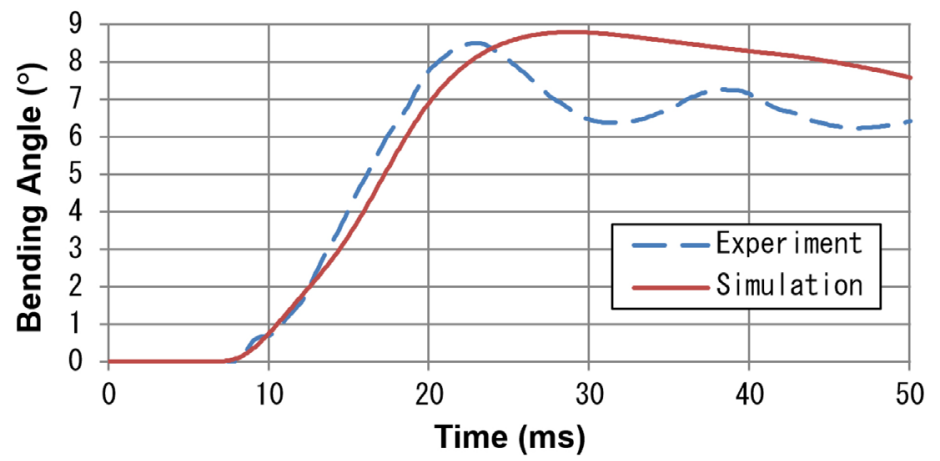

(c)

Figure 10. Comparison between experimented and predicted values of pedestrian injuries. 
between the experimented and the predicted injuries. Broken lines and solid lines indicate the experimented values and the predicted ones, respectively. With respect to tibia accelerations, the predicted ones agree quite well with the experimented ones in terms of a pattern of waveform and the difference of peak values within $7.4 \%$.

Regarding predicted shear displacements, the waveform as it rises up and general waveform are also similar to the experimented one. However, predicted peak value is $2 \mathrm{~mm}$ lager than the experimented. TRL legform impactor has the hydraulic damper near the ligaments, which is necessary to limit vibrations caused by the mass of the shear-spring system. It therefore follows that due to the damping effects the peak value is greatly suppressed and the waveform is attenuated with vibrations.

Concerned with bending angles, the waveform from the rise to the peak of the curve is almost similar each other and the difference of the peak value is 2.9 degrees equivalent to $3.4 \%$ as shown in Figure 10.

Three waveforms obtained by the TRL impactor experiment vibrate in common after their peaks. The period of the vibration is $10 \mathrm{~ms}$ which reflects the characteristic of the hydraulic damper of TRL legform impactor.

\section{Conclusion}

Shear forces were applied to the ligament in translational motion and the bending moments in rotational motion. Shear forces were described as the sum of viscous damping forces proportional to the deforming speed and spring forces proportional to the amounts of deformation, whereas bending moments were expressed as the sum of viscous damping moments proportional to the angular speed and rotational spring forces proportional to the bending angle. Damping coefficients and factors characteristic of the spring were determined experimentally and analytically. Differential equations were expressed in the form of a state equation and an output equation by MATLAB. Numerical solutions were obtained by a block diagram with Simulink. As a result, it was found that the predicted injuries agree quite well with their experimented data in terms of acceleration, displacement and the bending angle mentioned above.

\section{References}

[1] The Ministry of Land, Infrastructure and Transport, Government of Japan (JMLIT) (2011) Information on the Flexible Pedestrian Legform Impactor (Flex-PLI) from J-MLIT Research. The 7 th Meeting of the GRSP Informal Group on Pedestrian Safety (INF GR PS), Paris, 28-30 September 2004.

http://www.unece.org/trans/main/wp29/meeting_docs_grsp.html

[2] International Automobile Manufacturers Organization OICA (2008) JAMA's Explanation on the Pedestrian Safety GTR Phase 2. The 148 th Meeting of the Technical Committee, Paris, 14-15 October 2008.

[3] Lawrence, G.J.L. and Hardy, B.J. (1998) Pedestrian Testing Using the EEVC Pedestrian Impactors. Proceedings of the 16 th ESV Conference, Windsor, 31 May-4 June 1998, Paper Number 98-S10-O-03. 
[4] Leßmann, P. (BGS Boehme and Gehring) (2008) Die Impaktoren [The Impactors.] BGS Training Material, Bergisch Gladbach.

[5] UNECE (2011) Proposal for Amendments to Global Technical Regulation No. 9. The 47 th Session of the UNECE Working Party on Passive Safety (GRSP), Geneva, 17-21 May 2010, Document Number ECE/TRANS/WP.29/GRSP/2010/2). http://www.unece.org/trans/main/wp29/meeting_docs_grsp.html

[6] Gehring, D.-U. (BGS Boehme und Gehring), Leßmann, P. (BGS Boehme and Gehring) and Zander, O. (BASt) (2010) Enhanced Requirements for Front Ends Due to The New Test Regulations for Pedestrian Protection. Proceedings of the Crash. Tech 2010 Conference "Vehicle Safety 2020”, Leipzig, 13-14 April 2010.

[7] UNECE (2010) Global Technical Regulation No 9: Pedestrian Safety, Established in the Global Registry on 12 November 2008.

http://www.unece.org/trans/main/wp29/wp29wgs/wp29gen/wp29age.html 